\title{
Verification of Drogue Detection During Autonomous Aerial Refueling in a Simulatoin Environment
}

\author{
Oliver Ellis ${ }^{1}$ Umut Durak ${ }^{2}$ \\ ${ }^{1}$ Clausthal University of Technology, Institute of Informatics, 38678 Clausthal-Zellerfeld, Germany, oliver.ellis@dlr.de \\ ${ }^{2}$ German Aerospace Center (DLR), Institute of Flight Systems (FT), 38108 Braunschweig, Germany, umut.durak@dlr.de
}

\begin{abstract}
There are use cases that require to extend the airborne time for aircraft. Refueling is one challenge. Intuitively, an option is to do stopovers. Aircraft have to land, refuel and take off again. Another option is aerial refueling, avoiding to land. Autonomous aerial refueling is the refueling process conducted by aircraft in a solely automated manner. A pilot is not interfering during the execution. This process involves a taker aircraft carrying the fuel and a receiving follower aircraft. The follower enters in a leader-follower formation flight where the leader is the tanker. Then the tanker deploys a cone shaped drogue basket that rails from a flexible hose. The follower can insert a probe into the drogue basket to receive the fuel.
\end{abstract}

One challenge in autonomous aerial refueling is the automated location of the drogue basket. Object detection is a reasonable option for this task. Recent promising approaches come from the machine learning field. Convolutional Neural Networks (CNN) can be exploited to realize object detection. CNNs are specialized Deep Neural Networks (DNNs) that are comprised of several layers, namely convolutional layers, pooling layers and input and output layers. The input data is taken as matrices of pixels from images. With the output, the location of the drogue basket is predicted.

Like all airborne applications, safety is an essential aspect for aerial refueling. To meet the safety requirements for the automated docking, the detection quality of the object detection needs to be evaluated. However, it is harder than said. Flight tests are expensive and the effort intense. It is almost impossible to provide a broad enough coverage to gain confidence. Here the simulated testing presents a valuable lead.

A challenge is to create a synthetic scenario that is realistic enough and offers a sufficient number of features and variations. Modern game engines with photorealistic rendering capabilities have been investigated as options. Pursuing this idea, Unity 3D is applied. To render volumetric clouds and to obtain realistic weather effects, Unity is extended with the real time weather renderer trueSky. Hereby realistic images for aerial refuelling scenarios under different conditions are generated (e.g. overcast and precipitation).

It is a combinatorial problem to create enough test cases because of different types of overcast and weather effects and positional factors. The ontology framework System Entity Structure (SES) is utilized to cope with this issue. SESs are used to represent families of systems in simulation environments. This presentation proposes to construct an SES that represent a set of aerial refueling scenarios that are defined by a set of factors (overcast, precipitation, light, relative position and motion). A unique scenario is then derived by pruning (reducing the SES), obtaining a Pruned Entity Structure (PES). To only obtain meaningful scenarios, semantic constraints are applied. For example, if the sky is blue, it cannot rain. Such scenarios are the test cases.

In the simulation the specified test cases are loaded into the context and executed consecutively. During the process image files along with annotation data are generated.

To evaluate the results of the detection the Intersection over Union (IoU) method is used. Here the results are compared against a ground truth (a set of for this purpose prepared annotation data). In such way we obtain for each generated annotation data entry that we categorise with the labels true positive, false positive and false negative. True positive means that the drogue basket is detected with a sufficient accuracy. A false positive case means that detection is not as accurate. If we have a false negative case the CNN detected one or more drogue baskets at the wrong location in an image.

Thus, a desired result would be when above all due the true positives outweigh the other two categories by a large extend. In this case it can be summarised that the simulation based approach for aerial refueling has shown to be promising. 